\title{
Magnetic microgels for drug targeting applications: physical- chemical properties and cytotoxicity evaluation
}

\author{
Rodica Turcu ${ }^{\mathrm{a}}$, Izabell Craciunescu ${ }^{\mathrm{a}}$, Vasil M. Garamus ${ }^{\mathrm{b}}$, Christina Janko ${ }^{\mathrm{c}}$, Stefan Lyer ${ }^{\mathrm{c}}$, Rainer \\ Tietze $^{\mathrm{c}}$, Christoph Alexiou ${ }^{\mathrm{c}}$, Ladislau Vekas ${ }^{\mathrm{d}}$ \\ ${ }^{a}$ National Institute for Research and Development of Isotopic and Molecular Technologies, \\ 400293 Cluj-Napoca, Romania \\ ${ }^{b}$ Helmholtz-Zentrum Geesthacht, Zentrum für Material- und Küstenforschung GmbH, 21502 \\ Geesthacht, Germany \\ ${ }^{c}$ ENT-Department, Else Kröner-Fresenius Stiftung-Professorship, Section for Experimental \\ Oncology and Nanomedicine (SEON), University Hospital Erlangen, Germany \\ ${ }^{d}$ Romanian Academy-Timisoara Branch, CFATR, Lab. Magnetic Fluids, 300223 Timisoara, \\ Romania
}

\begin{abstract}
Magnetoresponsive microgels with high saturation magnetization values have been obtained by a strategy based on the miniemulsion method using high colloidal stability organic carrier ferrofluid as primary material. Hydrophobic nanoparticles $\mathrm{Fe}_{3} \mathrm{O}_{4}$ /oleic acid are densely packed into well-defined spherical nanoparticle clusters coated with polymers with sizes in the range 40$350 \mathrm{~nm}$. Physical-chemical characteristics of magnetic microgels were investigated by TEM, SAXS, XPS and VSM measurements with the focus on the structure-properties relationship. The impact of magnetic microgels loaded with anticancer drug mitoxantrone (MTO) on the nonadherent human $\mathrm{T}$ cell leukemia line Jurkat was investigated in multiparameter flow cytometry. We showed that both MTO and microgel-loaded MTO penetrate into cells and both induce apoptosis and later secondary necrosis in a time- and dose dependent manner. In contrast, microgels without MTO are not cytotoxic in the corresponding concentrations. Our results show that MTO-loaded microgels are promising structures for application in magnetic drug targeting.
\end{abstract}

Keywords: magnetic microgel, iron oxide nanoparticles, cytotoxicity, targeted drug delivery, Xray Photoelectron Spectroscopy, Small Angle X-ray Scattering

Corresponding authors: Rodica Turcu, National Institute for Research and Development of Isotopic and Molecular Technologies, 65-103 Donath Str., RO-400293 Cluj-Napoca, Romania, Phone: +40 264 584037, E-mail: rodica.turcu@itim-cj.ro; Ladislau Vekas, Romanian AcademyTimisoara Branch, CFATR, Lab. Magnetic Fluids, Mihai Viteazul Str. 24, RO-300223

Timisoara, Romania, E-mail: vekas@acad-tim.tm.edu.ro 


\section{Introduction}

Magnetic nanoparticle systems are receiving continuously increasing interest in the biomedical field for diagnosis and treatment $[1,2,3,4]$ due to their multiple functionalities as MRI contrast agents, magnetic hyperthermia treatments and magnetically guided drug delivery [5-7]. Superparamagnetic nanoparticles embedded in a polymer matrix [8], in particular in microgels, are highly promising magnetic carriers which offer several benefits concerning encapsulation of therapeutics, multivalency for bioconjugation, as well as mechanical and chemical stability in the specific bio-environment. The high magnetic moment of the functionalized carriers is among the most important requirements for successful applications in biomedicine, in particular for magnetic targeting [9]. Magnetic nanoparticle clusters in a polymer shell, summing up the magnetic moments of individual nanoparticles, were obtained by in situ coprecipitation of magnetic nanoparticles in microgels as microreactors $[10,11]$ and also by strongly polar solvent induced destabilization of a ferrofluid [12]. The controlled clusterization of magnetic nanoparticles, followed by encapsulation of the densely packed magnetic core in a polymer shell proved to be a facile and reproducible procedure when using the well-established ferrofluid technology and oil-in-water miniemulsion procedure $[13,14,15]$.

In this paper we present the oil-in-water miniemulsion based clustering process of surface coated magnetic nanoparticles, followed by entrapping the close packed magnetic core into the functional polymer shell. The efficient control of the evaporation induced self-assembly of magnetic nanoparticles, while keeping the superparamagnetic behavior of the resulted clusters was possible by starting with individually dispersed surfactant coated MNPs in a volatile organic solvent, i.e. from a highly stable ferrofluid. The structure, size characteristics and chemical composition were determined by transmission electron microscopy (TEM), Small Angle X-ray Scattering (SAXS) and X-ray Photoelectron Spectroscopy (XPS) respectively, and the magnetic properties were investigated by vibrating sample magnetometer (VSM). The anticancer drug loading and multiparameter flow cytometry analyses finally reveal the promising characteristics of the obtained magnetic microgel particles for drug targeting applications.

\section{Experimental section}

\subsection{Synthesis of magnetic microgels}


The magnetic microgels were obtained using a two steps synthesis procedure [16]: (i) the preparation of magnetic nanoparticle clusters (NPC) by oil-in-water miniemulsion technique [15, 17]; (ii) the coating of NPC with cross-linked polymer shells such as poly(Nisopropylacrylamide) (M-pNIPA) or poly(N-isopropylacrylamide)-polyacrylic acid (M-pNIPApAAc). The first step involved the emulsification of toluene based ferrofluid, containing $\mathrm{Fe}_{3} \mathrm{O}_{4}$ nanoparticles coated with a hydrophobic monolayer of oleic acid, in aqueous solution with sodium dodecyl sulphate (SDS) as surfactant. Any excess (free) oleic acid was eliminated from the initial ferrofluid. The mixture was treated ultrasonically to obtain small stable droplets of ferrofluid. An UP400S Compact Ultrasonic processor (100 W, 24 kHz; Hielscher Ultrasonics $\mathrm{GmbH}$ ) with PC control, sonotrode made of titanium was used to obtain the magnetic miniemulsion. The as prepared magnetic miniemulsion was heated to $100^{\circ} \mathrm{C}$ to remove the toluene and then was carefully washed several times with methanol-water mixture, magnetically separated and redispersed in water.

In the second step, the NPC coated with SDS were coated either with one polymer shell pNIPA or with two shells pNIPA-pAAc using layer by layer free radical polymerization. In a typical synthesis procedure, the aqueous solution containing NPC, the monomer (NIPA or AAc) and the cross-linker N,N-bisacrylamide (BIS) was stirred for 10 minutes, after that the oxidant ammonium persulfate (APS) was added to start the polymerization. The reaction mixture was kept under argon atmosphere at temperature $70^{\circ} \mathrm{C}$ and vigorous stirring. The as prepared magnetic microgel was precipitated using excess of acetone, washing several times to remove the unreacted products and redispersed in water.

\subsection{Characterization methods}

Transmission electron microscopy was carried out on a JEOL 1010 microscope to investigate the morphology of NPC and magnetic microgels. Structural investigations of NPC and microgels were performed by SAXS measurements using synchrotron radiation at the P12 BioSAXS facility with high brilliance X-ray beam (PETRA III storage ring - EMBL/DESY Hamburg). The beamline is optimized for solution scattering experiments and allows the investigations of water based suspensions of magnetic microgels. X-ray Photoelectron Spectroscopy was used to determine the surface chemical composition of NPC and microgels. XPS spectra were collected on an XPS spectrometer SPECS equipped with a dual-anode X-ray source Al/Mg, a PHOIBOS 
150 2D CCD hemispherical energy analyzer, a multi-channeltron detector with vacuum maintained at 1 x 10-9 Torr using AlK $\alpha$ X-ray source (1486.6 eV) operated at 200W. The particle suspension was dried on the indium foil to allow the XPS measurements. XPS data analysis and curve fitting was performed using CasaXPS software with a Gaussian-Lorentzian product function and a non-linear Shirley background substraction. The static magnetization of the samples was measured by means of vibrating sample magnetometry at room temperature using an ADE Technologies VSM 880 magnetometer.

\subsection{Cytotoxicity experiments}

UV-B sterilized microgels were incubated with mitoxantrone (MTO) for $96 \mathrm{~h}$. The effective loading of nanoparticles with MTO was calculated from the measurements of unbound MTO in the supernatant by an established HPLC method [18].

For assessment of toxicity we employed the non-adherent human T cell leukemia cell line Jurkat (DSMZ ACC 282). Cell culture was performed at $37^{\circ} \mathrm{C}$ and $5 \% \mathrm{CO}_{2}$ in RPMI 1640 medium supplemented with $10 \%$ Fetal Calf Serum (FCS), $1 \%$ glutamine, $1 \%$ penicillin-streptomycin (all from Invitrogen Life Technologies, Karlsruhe, Germany), and 1\% HEPES (10 mM, pH 7.2) (Merck KGaA,Darmstadt, Germany). For the experiments, the cells were adjusted to a density of $2 \times 10^{5}$ cells $/ \mathrm{ml}$ in cell culture media. $1 \mathrm{ml}$ of the cell suspensions were seeded into 48 well plates (Greiner Bioone, Frickenhausen, Germany) and incubated with unloaded and MTO loaded microgels. Untreated cells and cells treated with soluble MTO served as controls. The experiments were performed in triplicates. After $24 \mathrm{~h}$ and $48 \mathrm{~h}$ incubation $50 \mu \mathrm{l}$ aliquots of the cells were stained for $30 \mathrm{~min}$ at $4{ }^{\circ} \mathrm{C}$ with $250 \mu \mathrm{l}$ of a mixture of $20 \mu \mathrm{g} / \mathrm{ml}$ Propidium iodide (PI, Sigma-Aldrich, Taufkirchen, Germany), 10 nM DiIC1(5), $1 \mu \mathrm{g} / \mathrm{ml}$ Hoechst 33342 (Invitrogen Life Technologies, Karlsruhe, Germany) and $0.5 \mu \mathrm{g} / \mathrm{ml}$ FITC-labelled Annexin A5 (kindly provided by Internal Medicine 3, University Hospital Erlangen, Germany) in Ringer's solution (Baxter Healthcare, Zurich, Switzerland) as previously reported by Munoz et al. [19].

Finally, the cells were analyzed employing a Gallios flow cytometer (Beckman Coulter, Fullerton, CA, USA). Excitation for FITC and PI was at $488 \mathrm{~nm}$, the FITC fluorescence was recorded on FL1 sensor (525/38nm BP), the PI fluorescence on FL3 sensor (620/30 nm BP), the DiIC1(5) fluorescence was excited at $638 \mathrm{~nm}$ and recorded on FL6 sensor (675/20nm BP), and the Hoechst 33342 fluorescence was excited at $405 \mathrm{~nm}$ and recorded on FL9 sensor (430/40nm 
BP). The MTO fluorescence was excited at $638 \mathrm{~nm}$ and recorded by the FL-7 sensor (725/20 nm BP). Electronic compensation was used to eliminate bleed through fluorescence. Data analysis was performed with Kaluza software (Beckman Coulter, Fullerton, CA, USA).

\section{Results and discussion}

\subsection{Morphology and structure of magnetic microgels}

Well defined near spherical NPC have been obtained by oil-in-water miniemulsion method. Hydrophobic nanoparticles, $\mathrm{Fe}_{3} \mathrm{O}_{4}$ coated with oleic acid from the ferrofluid are densely packed into spherical clusters stabilized with SDS, as shown in Fig. 1. The aggregation observed in Fig. 1 is caused by the preparation of the sample for TEM investigation. The analysis of the TEM images enabled the determination of the diameters distributions of the nanoparticle clusters. As shown in the inset in Fig. 1, NPC have sizes in the range of 40-350 nm. Similar morphology of magnetic nanoparticle clusters prepared in different conditions has been reported in the literature $[14,15,20,21]$. The TEM images of the magnetic microgels M-pNIPA and M-pNIPA-pAAc presented in Fig.2, evidence that the spherical shape of NPC is preserved after polymer coating. The quasi-spherical shape of clusters and microgels resides mainly in the fact that the anisotropic dipolar interactions between individual magnetic nanoparticles are well-screened by the surfactant coating in the original ferrofluid and also in the prepared clusters, i.e. at close packing [22].

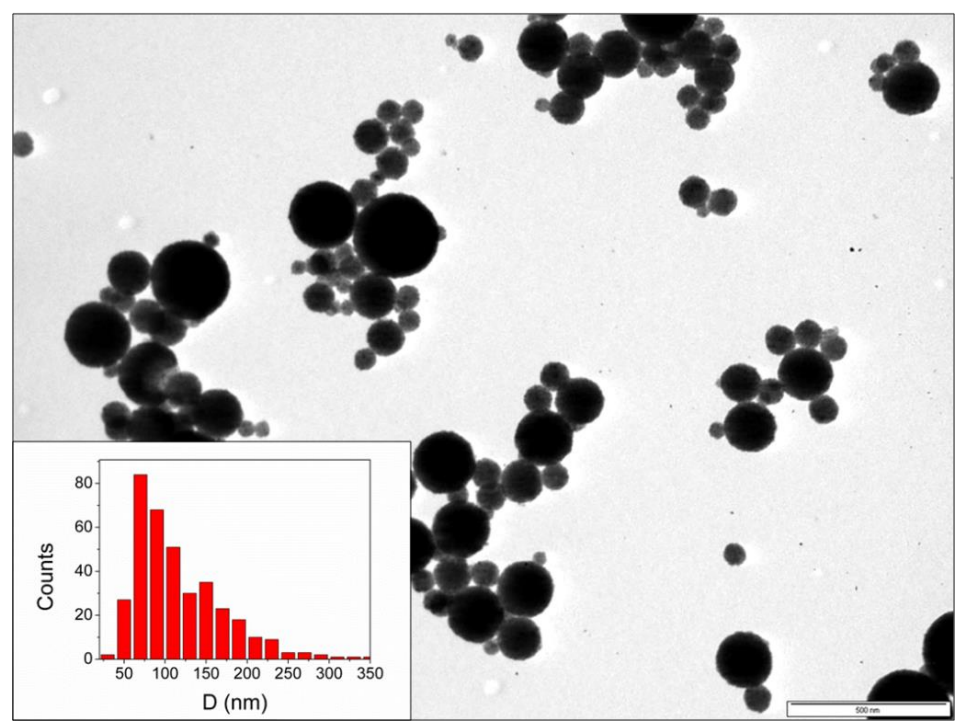

Fig. 1. TEM image of magnetic nanoparticle clusters stabilized with SDS (the bar is $500 \mathrm{~nm}$ ). Inset: the diameters distribution. 

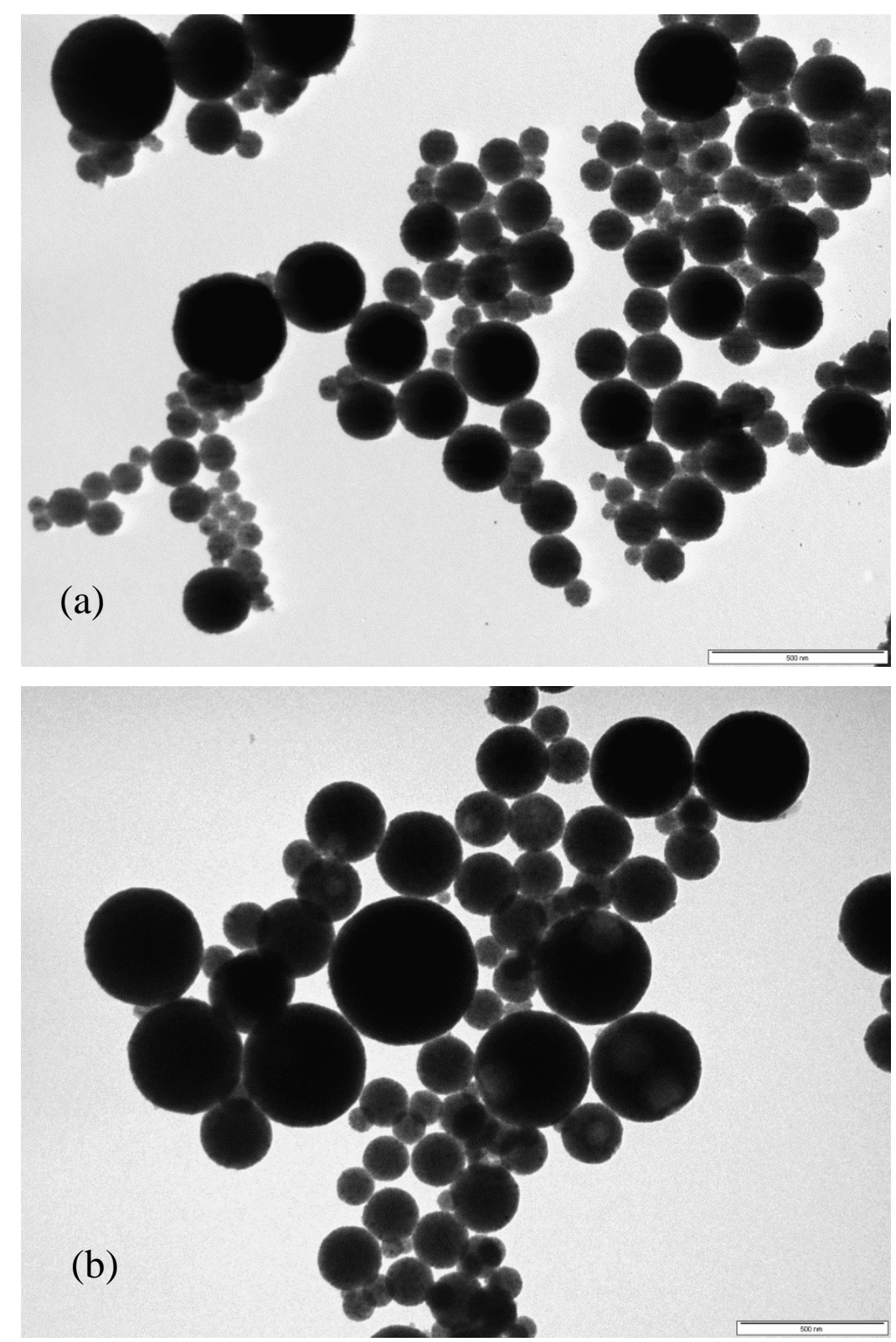

Fig. 2. TEM images of magnetic microgels: (a) M-pNIPA (the bar is $500 \mathrm{~nm}$ ); (b) M-pNIPApAAc (the bar is $500 \mathrm{~nm}$ ).

SAXS experiments were performed in aqueous solutions with variation of overall concentration from 0.1 to 0.8 wt. \%. After normalization to concentration the scattering patterns show negligible differences. It points to low interaction among NPC aggregates and their high stability. Due to much higher X-ray contrast for iron oxide/water compared with polymer \& surfactant/water the observed scattering intensities are from iron oxide nanoparticles. Examples 
of SAXS curves are presented in Fig. 3 which are typical for systems of objects with different length scales. Measured interval of scattering vector q reflects the structural information for objects in interval from 2 to $100 \mathrm{~nm}$. The scattering intensities do not reach a plateau at lowest $\mathrm{q}$ (0.06-0.3 $\left.\mathrm{nm}^{-1}\right)$ interval and show power law behavior i.e., $\mathrm{I} \sim \mathrm{q}^{-\alpha}$ which support the TEM data which show the presence of NPC of size more than $100 \mathrm{~nm}$. At an intermediate q range of $0.3-1$ $\mathrm{nm}^{-1}$ the plateau is observed which points to the presence of subunits of these large particles (or separated smaller nanoparticles, NP) in the interval of 1-10 nm. Taking these considerations into account the SAXS data have been analyzed by the Indirect Fourier Transformation (IFT) method [23], in the version given in [24] with trace scattering of larger particles in the power law form. By this analysis it is possible to get information on the surface or volume organization of large aggregates and also on the size and distribution of its subunits. IFT is a model-independent approach requiring only a minimum of preliminary information for analysis, i.e., the maximum dimensions of the objects $\left(\mathrm{D}_{\max }\right)$ via pair distance distribution function $\mathrm{p}(\mathrm{r})$ assuming that the scattering structures are 3D objects. A significantly better fit $\left(\chi^{2}\right.$ reduction 50\%) of scattering data was possible by considering the excluded volume repulsion between smaller aggregates, as well as by assuming the radius of hard sphere interaction equal to the size of particles and taking the excluded volume fraction equal to 0.1. The obtained $p(r)$ functions are shown in Fig. 4 and parameters of system such as $\alpha, D_{\max }$ and mean value of radius of gyration, $\mathrm{R}_{\mathrm{g}}$ of subunits are summarized in Table 1.

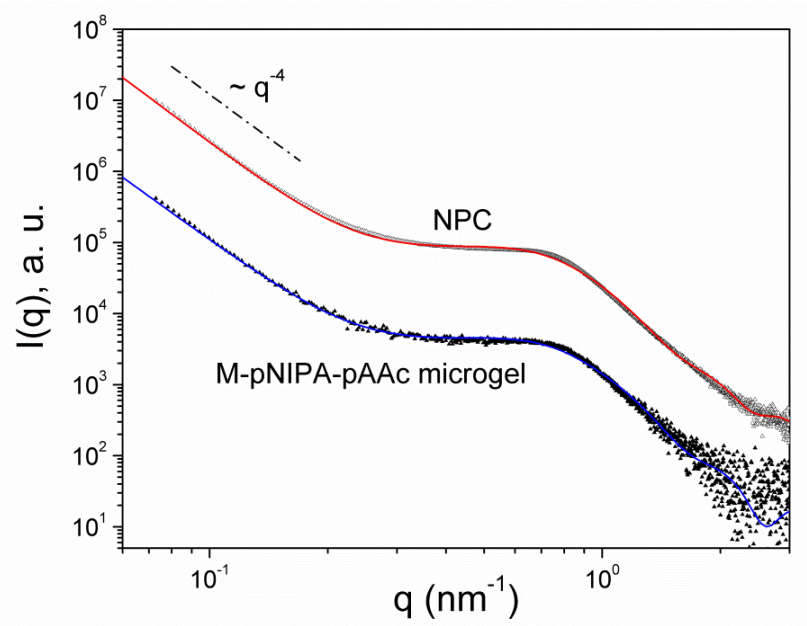

Fig. 3. SAXS curves for magnetic nanoparticle clusters (NPC, c=0.8 wt.\%) and microgel sample M-pNIPA-pAAc (c=0.1 wt. \%) 
In the above analysis the value of $D_{\max }$ has been varied to fit the data and to get the shape of $p(r)$ function which smoothly goes to zero at maximal distance; in some cases the $\mathrm{p}(\mathrm{r})$ function has been forced to zero (for exemple, sample M-pNIPA, Fig. 4).

\section{Table 1}

Results of IFT analysis with trace scattering of NPC in power law form: $\alpha$ - slope in the low q part $\left(\mathrm{q}<0.2 \mathrm{~nm}^{-1}\right), \mathrm{D}_{\max }-$ maximal distance within NPC required and $\mathrm{R}_{\mathrm{g}}$ - mean value of radius of gyration of NP and statistical error of determination of mean value.

\begin{tabular}{cccc}
\hline Sample & $\boldsymbol{\alpha}, \pm \mathbf{0 . 0 5}$ & $\boldsymbol{D}_{\boldsymbol{m a x}}, \boldsymbol{n m}$ & $\boldsymbol{R}_{\boldsymbol{g}}, \boldsymbol{n m}$ \\
\hline NPC & 4.00 & 6 & $2.1 \pm 0.1$ \\
\hline M-pNIPA-pAAc & 4.15 & 6.5 & $2.1 \pm 0.1$ \\
\hline MNIPA 325 & 4.55 & 7 & $2.5 \pm 0.1$ \\
\hline
\end{tabular}

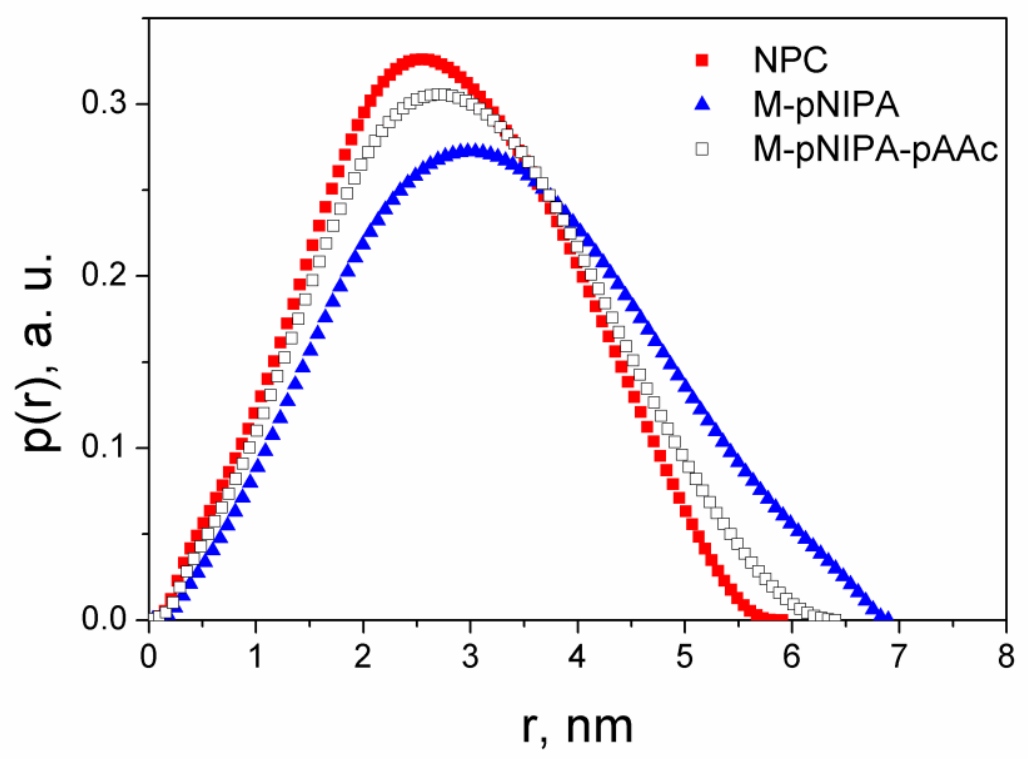

Fig. 4. Shape of $p(r)$ function of subunits (NP) depending on type of coating.

All $\mathrm{p}(\mathrm{r})$ functions exhibit nearly symmetrical shape which supports that NP are nanospheres. For all of NPC a small repulsion between NP is observed, which is connected with the nature of interaction potential (absence of electrical charge) and concentration of NP in larger aggregates. 
The main difference is observed in the structure of interface of larger aggregates, i.e. the change of slope from 4 to 4.5 shows the change of interface from smooth and sharp to diffusive interface [25]. In this case the scattering profile of interface is written via:

$$
\rho(r)=\left\{\begin{array}{l}
\rho, 0<r<r_{\max }-a \\
\left(\frac{r_{\max }-r}{a}\right)^{\beta}, r_{\text {max }}-a<r<r_{\text {max }} \\
0, r>r_{\text {max }}
\end{array}\right.
$$

In this equation $r_{\max }$ is the radius of aggregates, $r$ - the distance from centre, $a$ - the thickness of developed layer and $\beta=(\alpha-4) / 2$. In Fig. 5 we have plotted the possible sketch of interface profile for different gel composition with assumption of thickness equal to the diameter of NP, i.e., $7 \mathrm{~nm}$ and size of large aggregates is equal to $200 \mathrm{~nm}$.

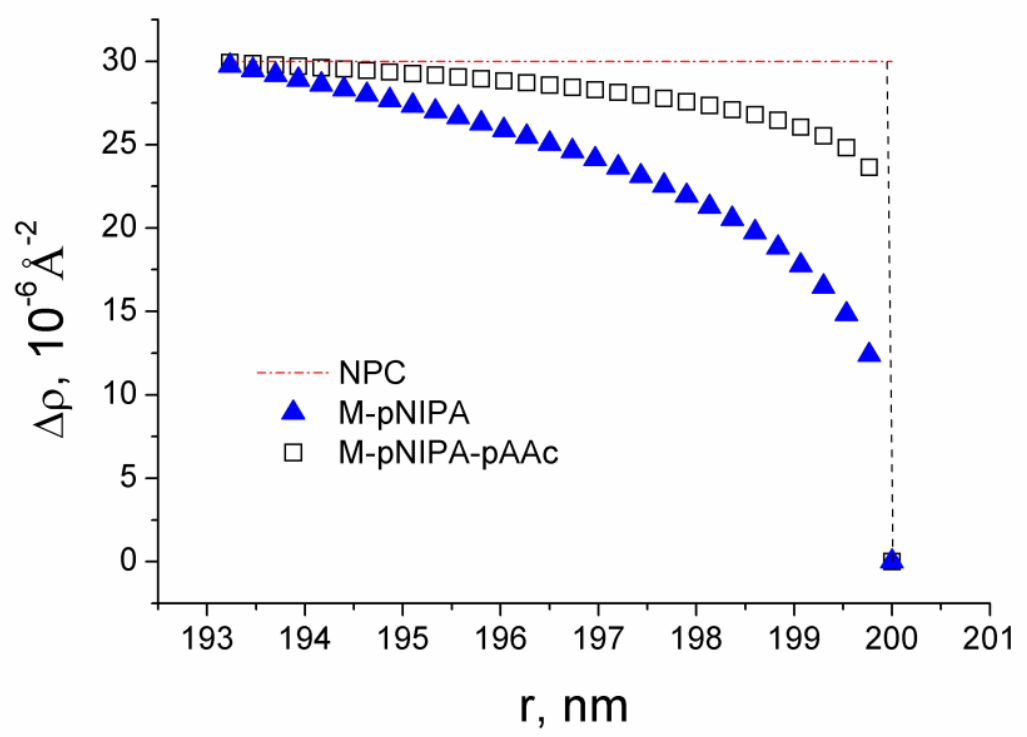

Fig. 5. The X-ray scattering profile of interface of large NPC depending on microgel composition.

SAXS data clearly show the formation of large NPC which consist of smaller NP and these NP are well separated and show repulsive interaction. The addition of polymer changes the interface of NPC from smooth and sharp to diffusive. 


\subsection{X-ray Photoelectron Spectroscopy}

XPS was employed to examine the composition as well as the chemical state of the atoms of the as-prepared magnetic microgels. The former information is inferred from the areas delimited by the photoelectron peaks and the latter one relates to the chemical shifts of the peaks with respect to the elemental state. Chemical shift information is a very powerful tool for functional group, chemical environment and oxidation state. Fig. 6 shows the high resolution XPS spectra of C1s, O1s, N1s, S2p, Fe2p core levels from M-pNIPA microgel. The successful formation of the magnetic microgel by coating of NPC stabilized with SDS with the cross linked pNIPA is evidenced by the characteristic peaks in XPS spectra from Fig. 6: (i) $\mathrm{C}-\mathrm{N}(285.8 \mathrm{eV}), \mathrm{N}-\mathrm{C}=\mathrm{O}$ $(288 \mathrm{eV})$ in $\mathrm{C} 1 \mathrm{~s}$ spectrum; $\mathrm{NH}(399.6 \mathrm{eV})$ in N1s spectrum; $\mathrm{C}=\mathrm{O}(531.5 \mathrm{eV})$ in $\mathrm{O} 1 \mathrm{~s}$ spectrum, which are specific for pNIPA and the crosslinker BIS; (ii) S2p spectrum containing the doublet $\mathrm{S} 2 \mathrm{p} 3 / 2$ and S2p1/2 at binding energies $168.5 \mathrm{eV}$ and $169.6 \mathrm{eV}$ respectively which correspond to sulfur atoms from sulfate group of SDS; (iii) the Fe $2 \mathrm{p}$ spectrum contains the doublet Fe 2p3/2 and $\mathrm{Fe} 2 \mathrm{p} 1 / 2$ and their satellites; each peak of the Fe2p spectrum can be deconvoluted into two components corresponding to $\mathrm{Fe}^{3+}$ and $\mathrm{Fe}^{2+}$ ions [26, 27]. The fitting parameters including the peaks positions, full width at half maximum (FWHM) and calculated atomic concentrations from peaks areas for the sample M-pNIPA are given in Table 2. From the data in the Table 2 we obtained the atomic concentrations ratio $\mathrm{C} / \mathrm{Fe}=3.12$ for the sample M-pNIPA.

\section{Table 2}

The fitting parameters of XPS spectra (peaks positions, FWHM and calculated atomic concentrations from peaks areas) for the sample M-pNIPA

\begin{tabular}{cccc}
\hline Peak name & Position $(\boldsymbol{e V})$ & FWHM $(\boldsymbol{e V})$ & $\begin{array}{c}\text { \% atomic } \\
\text { concentration }\end{array}$ \\
\hline $\mathrm{C} 1 \mathrm{~s}$ & 284.72 & 2.295 & 35.480 \\
\hline $\mathrm{C} 1 \mathrm{~s}$ & 285.75 & 2.595 & 7.093 \\
\hline $\mathrm{C} 1 \mathrm{~s}$ & 288 & 2.377 & 8.279 \\
\hline $\mathrm{O} 1 \mathrm{~s}$ & 529.72 & 1.898 & 6.254 \\
\hline $\mathrm{O} 1 \mathrm{~s}$ & 531.02 & 3.563 & 20.612 \\
\hline $\mathrm{N} 1 \mathrm{~s}$ & 399.64 & 2.341 & 4.190 \\
\hline $\mathrm{S} 2 \mathrm{p}$ & 168.50 & 2.28 & 0.909 \\
\hline $\mathrm{S} 2 \mathrm{p}$ & 169.64 & 2.233 & 0.889 \\
\hline $\mathrm{Fe}^{2+} 2 \mathrm{p} 3 / 2$ & 709.94 & 2.638 & 2.152 \\
\hline $\mathrm{Fe}^{3+} 2 \mathrm{p} 3 / 2$ & 711.55 & 3.613 & 3.429 \\
\hline
\end{tabular}




\begin{tabular}{cccc}
\hline $\mathrm{Fe} 2 \mathrm{p} 3 / 2$ satellite & 714.14 & 3.575 & 1.481 \\
\hline $\mathrm{Fe} 2 \mathrm{p} 3 / 2$ satellite & 717.91 & 5.600 & 1.411 \\
\hline $\mathrm{Fe}^{2+} 2 \mathrm{p} 1 / 2$ & 723.56 & 3.252 & 2.084 \\
\hline $\mathrm{Fe}^{3+} 2 \mathrm{p} 1 / 2$ & 725.07 & 4.997 & 3.320 \\
\hline $\mathrm{Fe} 2 \mathrm{p} 1 / 2$ satellite & 727.43 & 4.547 & 1.077 \\
\hline $\mathrm{Fe} 2 \mathrm{p} 1 / 2$ satellite & 732.39 & 5.312 & 1.342 \\
\hline
\end{tabular}
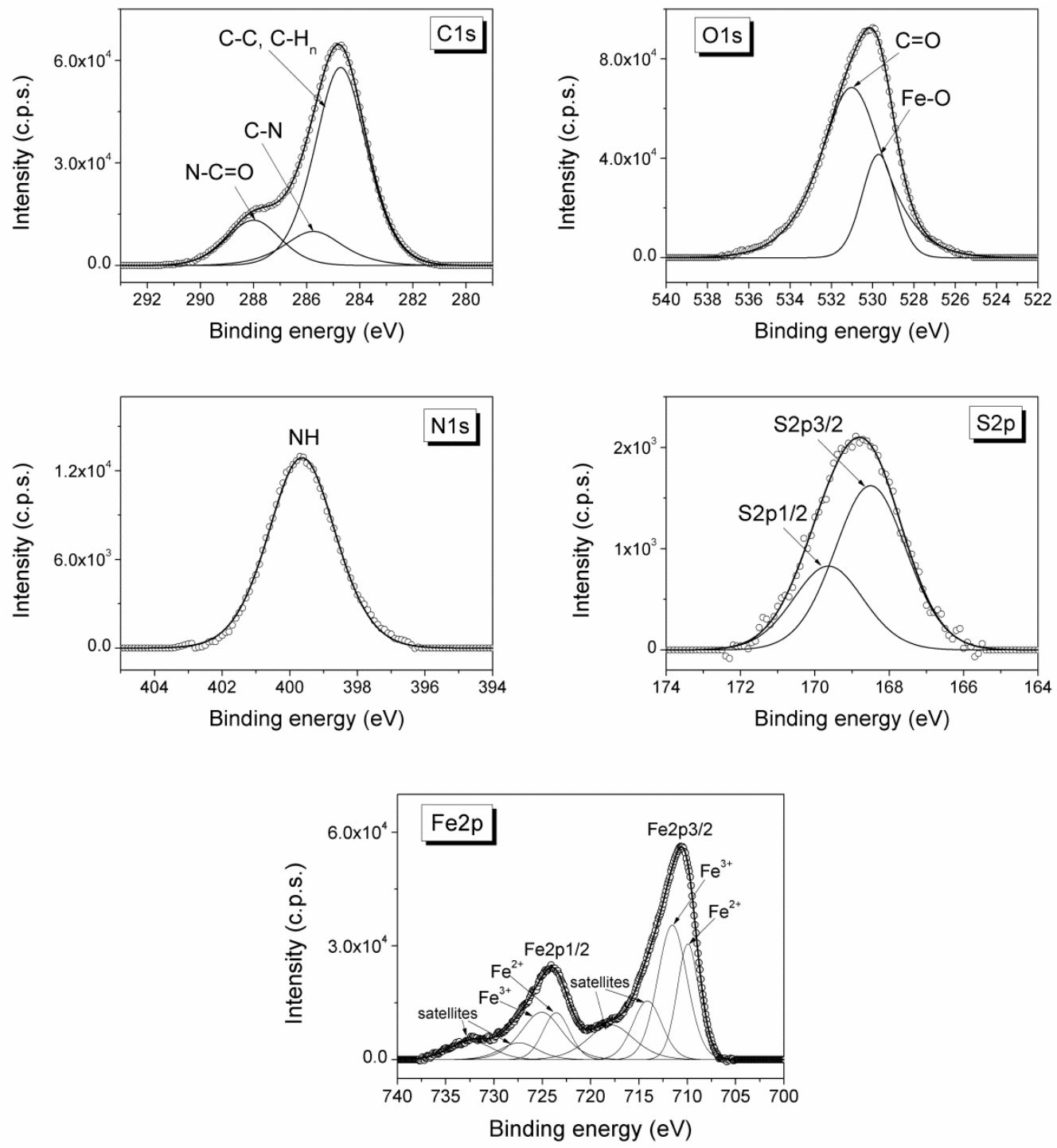

Fig. 6. High resolution XPS spectra of $\mathrm{C} 1 \mathrm{~s}, \mathrm{O} 1 \mathrm{~s}, \mathrm{~N} 1 \mathrm{~s}, \mathrm{~S} 2 \mathrm{p}, \mathrm{Fe} 2 \mathrm{p}$ core levels of M-pNIPA microgel.

In case of M-pNIPA-pAAc microgel the XPS spectra of N, S and Fe core-levels are similar to those of M-pNIPA and consequently are not shown. Relevant differences appear in C1s and O1s 
core-level spectra of M-pNIPA-pAAc (Fig. 7) in comparison with M-pNIPA (Fig. 6). The best fit of $\mathrm{C} 1 \mathrm{~s}$ spectrum from Fig. 7 contains four components assigned to $\mathrm{C}-\mathrm{C} / \mathrm{CH}(284.85 \mathrm{eV}), \mathrm{C}-\mathrm{N}$ $(285.75 \mathrm{eV}), \mathrm{N}-\mathrm{C}=\mathrm{O}(287.5 \mathrm{eV})$ and $\mathrm{O}-\mathrm{C}=\mathrm{O}(288.8 \mathrm{eV})$. The $\mathrm{O} 1 \mathrm{~s}$ spectrum shown in Fig. 7 was deconvoluted into three component peaks ascribed to Fe-O group from iron oxide (529.55 eV), $\mathrm{C}=\mathrm{O}(531.2 \mathrm{eV})$ and $\mathrm{O}-\mathrm{C}=\mathrm{O}(533 \mathrm{eV})$. The presence of the carboxyl groups on the surface of microgel evidence the formation of the polyacrylic layer. Table 3 shows the fitting parameters of XPS spectra for the sample M-pNIPA-pAAc. A higher value of atomic concentrations ratio C/Fe $=15.96$ was obtained for the sample M-pNIPA-pAAc as compared with M-pNIPA, as expected.
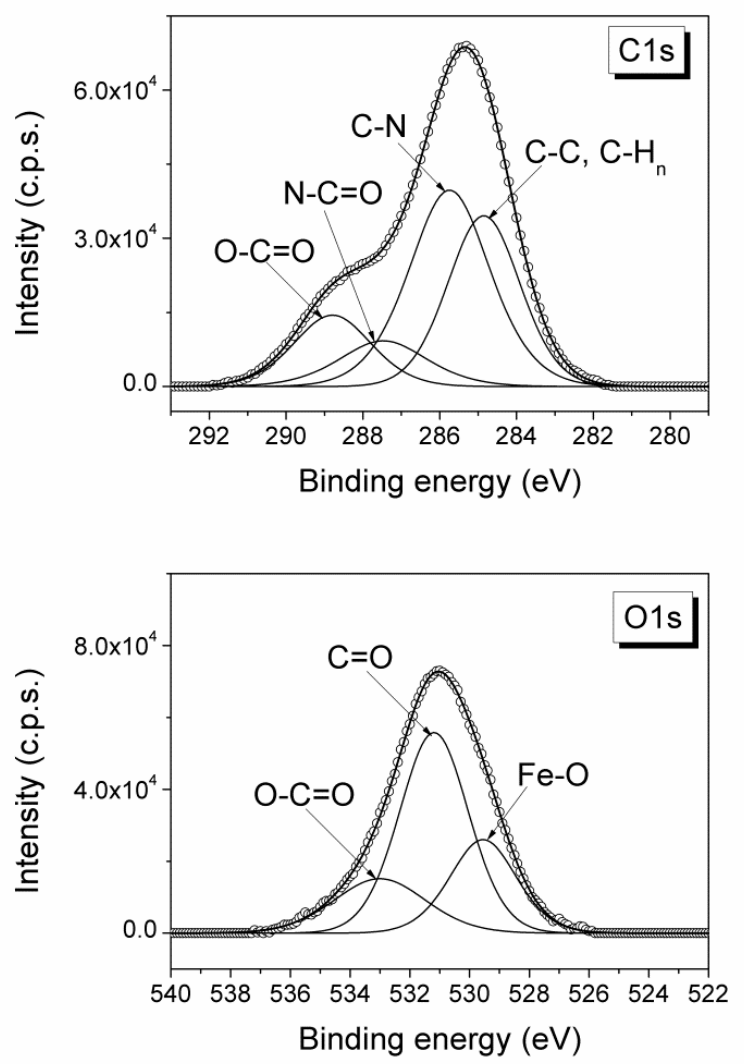

Fig. 7. High resolution XPS spectra of C1s and O1s core-levels of M-pNIPA-pAAc microgel. 


\section{Table 3}

The fitting parameters of XPS spectra (peaks positions, FWHM and calculated atomic concentrations from peaks areas) for the sample M-pNIPA-pAAc

\begin{tabular}{cccc}
\hline Peak name & Position $(\boldsymbol{e V})$ & $\boldsymbol{F W H M}(\boldsymbol{e V})$ & $\begin{array}{c}\text { \%o atomic } \\
\text { concentration }\end{array}$ \\
\hline $\mathrm{C} 1 \mathrm{~s}$ & 284.85 & 2.216 & 21.273 \\
\hline $\mathrm{C} 1 \mathrm{~s}$ & 285.75 & 2.471 & 27.948 \\
\hline $\mathrm{C} 1 \mathrm{~s}$ & 287.47 & 2.685 & 7.069 \\
\hline $\mathrm{O} 1 \mathrm{~s}$ & 288.80 & 2.381 & 9.647 \\
\hline $\mathrm{O} 1 \mathrm{~s}$ & 529.55 & 2.62 & 5.827 \\
\hline $\mathrm{O} 1 \mathrm{~s}$ & 531.19 & 2.719 & 12.815 \\
\hline $\mathrm{N} 1 \mathrm{~s}$ & 533.01 & 3.612 & 4.614 \\
\hline $\mathrm{S} 2 \mathrm{p}$ & 399.51 & 2.366 & 5.431 \\
\hline $\mathrm{S} 2 \mathrm{p}$ & 168.68 & 2.521 & 0.631 \\
\hline $\mathrm{Fe}^{2+} 2 \mathrm{p} 3 / 2$ & 169.62 & 2.413 & 0.617 \\
\hline $\mathrm{Fe}$ & 709.53 & 2.426 & 0.555 \\
\hline $\mathrm{Fe} 2 \mathrm{p} 3 / 2 \mathrm{satellite}$ & 711.12 & 3.31 & 0.983 \\
\hline $\mathrm{Fe} 2 \mathrm{p} 3 / 2 \mathrm{satellite}$ & 713.59 & 2.978 & 0.386 \\
\hline $\mathrm{Fe}^{2+} 2 \mathrm{p} 1 / 2$ & 716.20 & 5 & 0.283 \\
\hline $\mathrm{Fe}^{3+} 2 \mathrm{p} 1 / 2$ & 723.07 & 2.839 & 0.531 \\
\hline $\mathrm{Fe} 2 \mathrm{p} 1 / 2 \mathrm{satellite}$ & 725.13 & 4.5 & 0.939 \\
\hline $\mathrm{Fe} 2 \mathrm{p} 1 / 2 \mathrm{satellite}$ & 732.42 & 4.008 & 0.177 \\
\hline
\end{tabular}

\subsection{Magnetic properties}

The magnetic measurements were performed on dried samples of NPC stabilized with SDS and on the microgels obtained by polymer coating of NPC. Fig. 8 shows the magnetization versus magnetic field at room temperature of NPC and microgels M-pNIPA and M-pNIPA-pAAc. 


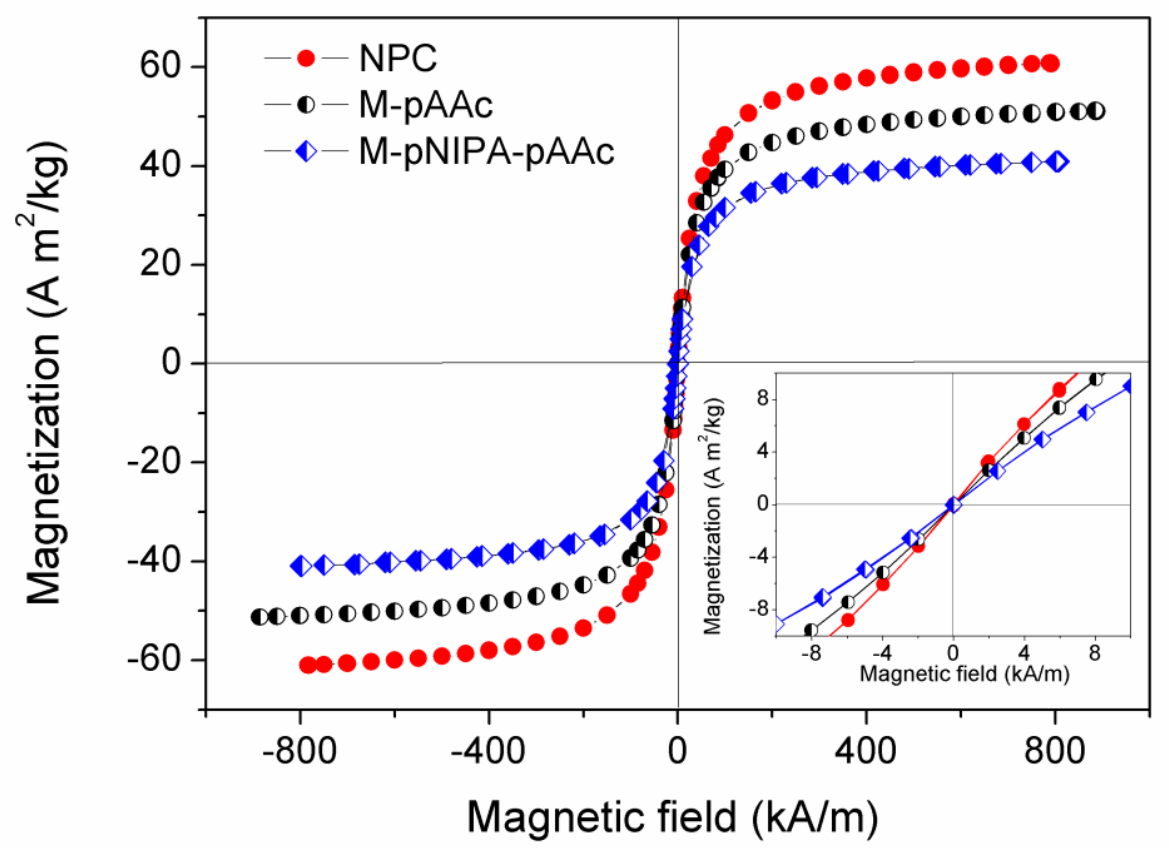

Fig. 8. Magnetization curves at room temperature of NPC and of the microgels M-pNIPA, MpNIPA-pAAc.

For all the samples the magnetization at room temperature does not show any hysteresis loop, being consistent with a superparamagnetic behavior, as expected for oleic acid-coated magnetite nanoparticles of small sizes (less than $10 \mathrm{~nm}$ ) from the ferrofluid used as primary material. Moreover, this fact indicates that the magnetite nanoparticles are still well separated in NPC and the magnetic dipole-dipole interactions between them are negligible. High saturation magnetization values were obtained for the nanoparticle clusters, $\mathrm{Ms}=64 \mathrm{~A} \mathrm{~m}^{2} / \mathrm{kg}$ and also for the magnetic microgels, $M_{S}=53 \mathrm{~A} \mathrm{~m}^{2} / \mathrm{kg}$ for M-pNIPA and $\mathrm{M}_{\mathrm{S}}=43 \mathrm{~A} \mathrm{~m}^{2} / \mathrm{kg}$ for M-pNIPApAAc, which are somewhat lower than the values measured for NPC, due to the influence of polymer coating (Fig.8).

\subsection{Cytotoxicity}

The binding capacity of the microgels was $35 \mu \mathrm{g}$ MTO/ $\mu \mathrm{g}$ Fe original substance for M-pNIPA and $37 \mu \mathrm{g} \mathrm{MTO} / \mu \mathrm{g}$ Fe original substance for M-pNIPA-pAAC as determined by HPLC analysis. For cytotoxicity assessment, Jurkat cells were incubated with unloaded microgels and MTO loaded microgels. Cells treated with fluid MTO and untreated cells served as positive and 
negative controls, respectively. The chemotherapeutic agent MTO has an inherent fluorescence; therefore its uptake by cells can be easily monitored by flow cytometry. Thus, cells treated with different concentrations of MTO showed a concentration dependent increase in their intracellular MTO fluorescence. Note that cells treated with fluid MTO have a stronger intracellular fluorescence compared to the MTO-microgels treated cells, indicating that fluid MTO is taken up better by cells than microgel bound MTO. Untreated cells merely showed autofluorescence (Fig. 9).

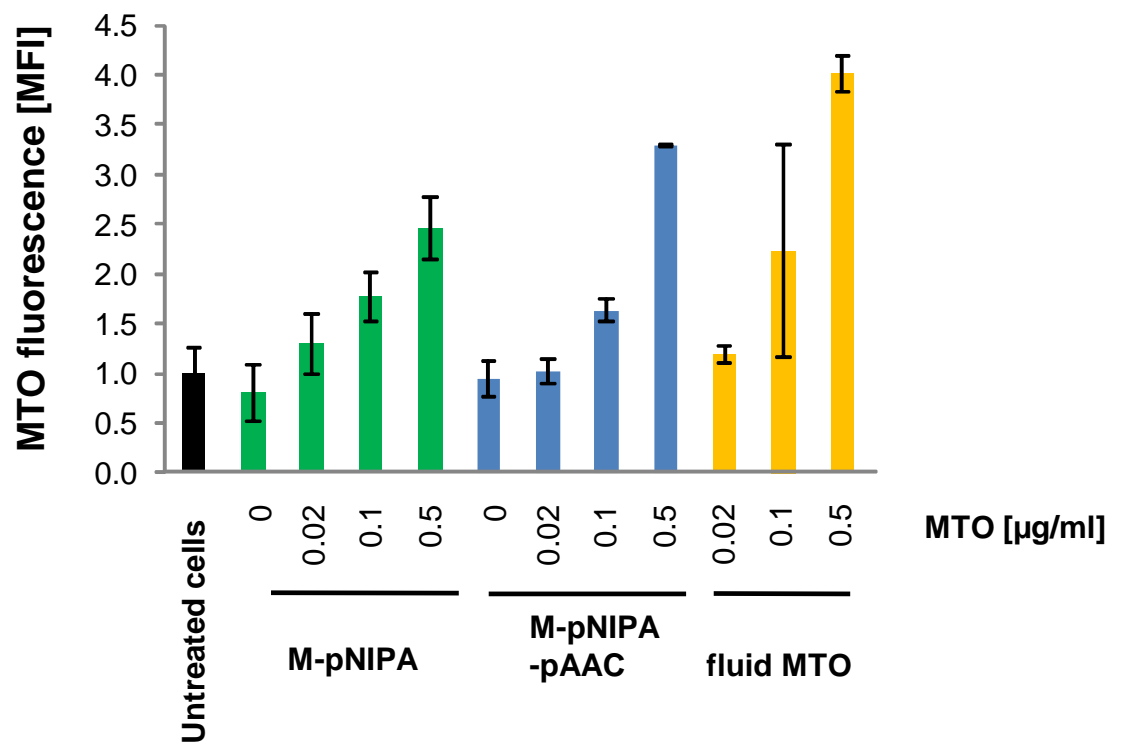

Fig. 9. Intracellular MTO fluorescences [MFI] of cells treated with MTO loaded microgels after $24 \mathrm{~h}$ of incubation. Shown are the mean values of triplicates.

Cell death induction by the cytotoxic drug MTO was monitored by DiIC1(5) staining and subsequent analysis of the cells in flow cytometry. DiIC1(5) fluorescence is emitted from healthy cells with polarized mitochondrial membrane. Decrease in DiIC(1)5 fluorescence indicates loss of mitochondrial membrane potential and cell death. Cells were analysed for cell death after $0 \mathrm{~h}$, $24 \mathrm{~h}$, and $48 \mathrm{~h}$ of incubation. To exclude that cytotoxic effects are mediated by the microgels themselves, unloaded microgels were used as controls (Fig. 10).

When we analysed the cell death pattern induced by MTO, we found that the fluid MTO and MTO loaded microgels both induce cell death in a time- and dose dependent manner. In the high MTO concentration $(0.5 \mu \mathrm{g} / \mathrm{ml})$ the cytotoxicity of fluid MTO and microgel loaded MTO is similar. In the low MTO concentrations $(0.02 \mu \mathrm{g} / \mathrm{ml})$ fluid MTO is more cytotoxic compared to 
microgel loaded MTO, probably because of a delayed bioavailibility of microgel bound MTO as already shown by the analysis of intracellular MTO fluorescence (Fig. 9). In contrast, unloaded microgels are not cytotoxic in the corresponding Fe concentrations.
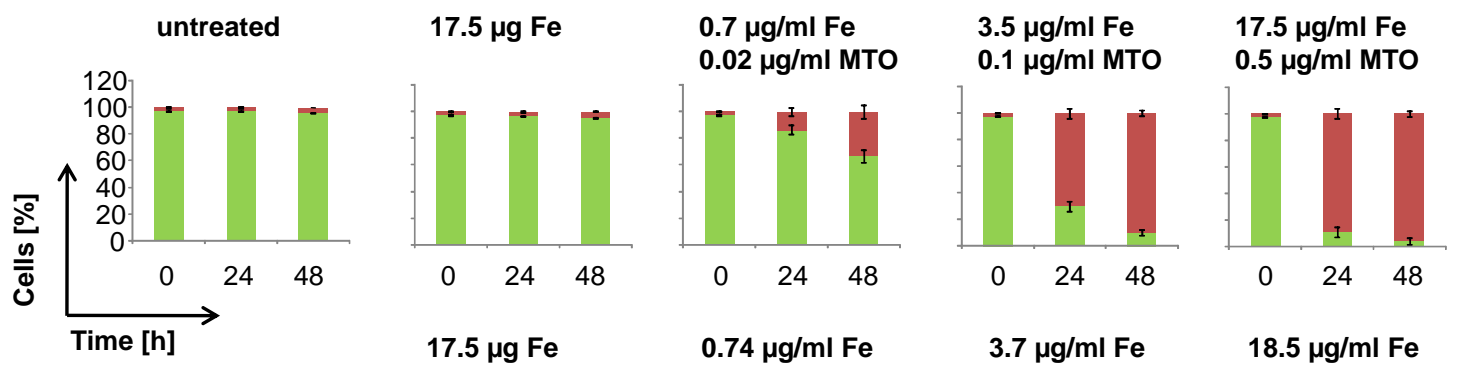

M-pNIPA
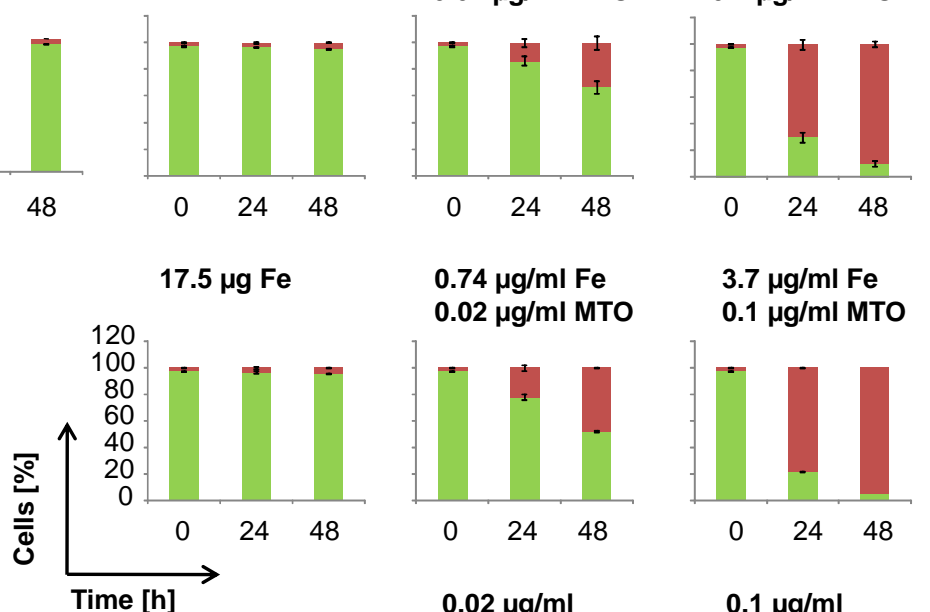

$18.5 \mu \mathrm{g} / \mathrm{ml} \mathrm{Fe}$
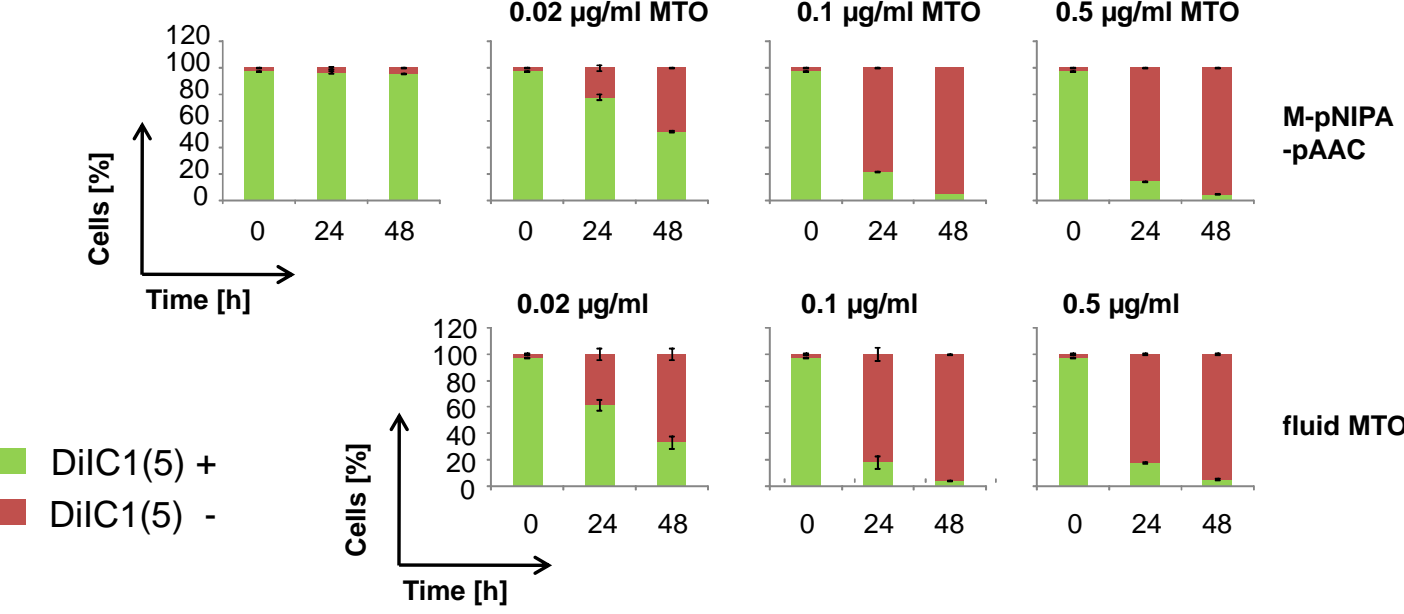

fluid MTO

Fig. 10. Mitochondrial membrane potential of Jurkat cells treated with MTO loaded microgels. Shown are the mean values of triplicates.

The results of the DiIC1(5) assay were also confirmed by Annexin (Ax) V-Fitc/Propidiuimiodid (PI) staining (Fig. 11). Ax V-Fitc binds to phosphatidylserine, a plasma membrane lipid, found on the inner leaflet of the cell membrane in viable cells. In apoptosis, phosphatidylserine is translocated to the outer leaflet and can be bound by Ax V-Fitc. The plasma membrane impermeable dye PI is added to distinguish between apoptosis and necrosis. If the cells have a disrupted plasma membrane, PI is able to penetrate the plasma membrane and to intercalate into the DNA, thus serving as marker for necrosis. Ax-PI- cells thus are assumed to be viable, Ax+PIcells are apoptotic, and $\mathrm{Ax}+\mathrm{PI}+$ cells are necrotic.

In line with the DiIC1(5) staining, the AxPI staining shows that MTO and MTO loaded microgels induce cell death in a time and dose dependent manner, whereas fluid MTO is more cytotoxic at low concentration $(0.02 \mu \mathrm{g} / \mathrm{ml}$ MTO $)$. Note that most of the cells treated with 0.5 
$\mu \mathrm{g} / \mathrm{ml} \mathrm{MTO}$ are Ax+PI- at 48h observation time, which is not apoptosis in this case; instead it results from degraded DNA in late secondary necrosis.

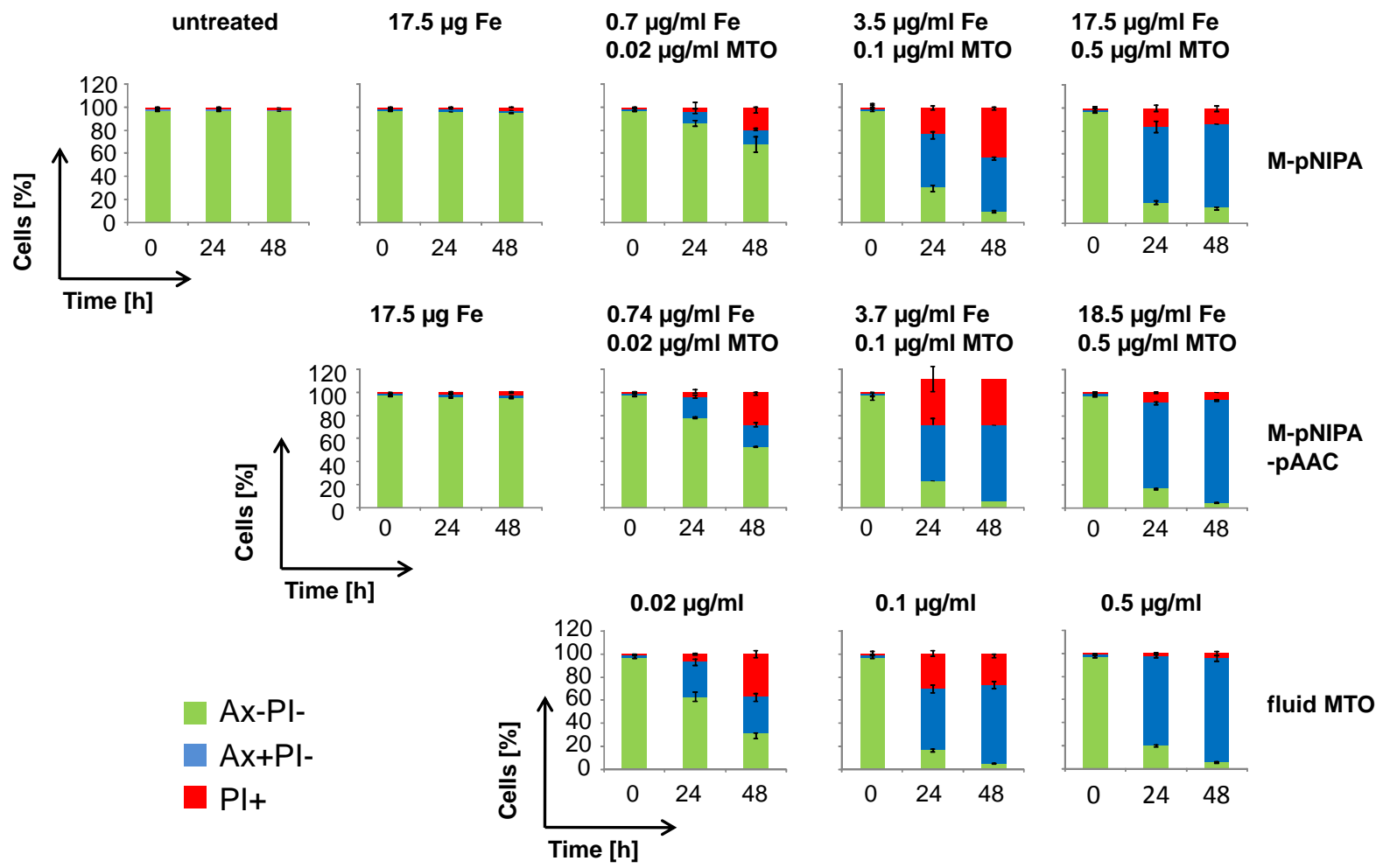

Fig. 11. Phosphatidylserine exposure and plasma membrane integrity of Jurkat cells treated with MTO loaded microgels. Shown are the mean values of triplicates.

\section{Conclusions}

Low boiling point and high colloidal stability ferrofluids were used for the preparation of magnetic microgels designed for application in nanomedicine. Clusters of magnetite nanoparticles were prepared in a reproducible fashion using the miniemulsion method. The coating of the clusters of magnetite nanoparticles with polymers results in magnetic microgels with a core-shell structure, high magnetization $\left(43-53 \mathrm{Am}^{2} / \mathrm{kg}\right.$ ), superparamagnetic behaviour and good stability as water-based suspensions. SAXS data clearly show the formation of large NPC which consist of smaller NP and these NP are well separated and show repulsive interaction. The addition of polymer changes the interface of NPC from smooth and sharp to diffusive. The successful formation of the magnetic microgel by coating of NPC stabilized with SDS with the cross linked pNIPA is evidenced by the characteristic peaks in XPS spectra. 
Relevant differences were evidenced in C1s and O1s core-level spectra of M-pNIPA-pAAc in comparison with M-pNIPA. The magnetic microgel synthesis procedure involving advanced ferrofluid manufacturing and miniemulsion procedure is facile and well-controlled to be applied for up-scaled synthesis of magnetic microgels.

The toxicity tests using the human $\mathrm{T}$ cell leukemia cell line Jurkat show that the magnetic microgels alone are not cytotoxic in the corresponding Fe concentrations. As recently as the microgels are loaded with the anticancer drug MTO, the microgels become cytotoxic. Thus, fluid MTO and MTO-loaded microgels both induce cell death in a time- and dose dependent manner. In high MTO concentrations the cytotoxicity of fluid MTO and microgel loaded MTO is similar; in low MTO concentrations $(0.02 \mu \mathrm{g} / \mathrm{ml})$ fluid MTO is more cytotoxic, probably because of a delayed bioavailibility of microgel bound MTO.

Our results show that the high magnetization magnetic microgels have promising applications as magnetic carriers for anticancer drug targeting.

\section{Acknowledgment}

This study was supported by the Bavarian Ministry for Environment and Consumer Protection (74-U8793-2012/7-35), the Cluster of Excellence Engineering of Advanced Materials (EAM), DFG (SPP1681, AL552/5-1) and BMBF (FKZ 01DS13012). We gratefully acknowledge the technical support from Clement Blanchet at the P12 BioSAXS beamline (EMBL/DESY, PETRA III). R. Turcu gratefully acknowledges the financial support from ROSEAL S.A, Odorheiu Secuiesc, Romania. We are indebted to Oana Marinica, PhD fellow and to Florica Balanean (University Politehnica Timisoara, Research Center for Engineering of Systems with Complex Fluids) for VSM measurements and for the toluene based magnetic fluid. Valuable discussions with Dr. V. Socoliuc (CFATR) are gratefully acknowledged. The work of L. Vekas was supported by the research program (2013-2015) of CFATR.

\section{References}

[1] S.R. Dave, X. Gao, Monodisperse magnetic nanoparticles for biodetection, imaging, and drug delivery: a versatile and evolving technology, Nanomedicine and Nanobiotechnology, 1 (2009) 583-609 
[2] M. Mahmoudi, S. Sant, B. Wang, S. Laurent, T. Sen, Superparamagnetic iron oxide nanoparticles (SPIONs): development, surface modification and applications in chemotherapy, Adv. Drug Deliv. Rev. 63 (2011) 24-46

[3] M. Szekeres, I. Y. Tóth, E. Illés, A. Hajdú, I. Zupkó, K. Farkas, G. Oszlánczi, L. Tiszlavicz, E. Tombácz, Chemical and Colloidal Stability of Carboxylated Core-Shell Magnetite Nanoparticles Designed for Biomedical Applications, Int. J. Mol. Sci., 14 (2013), 14550-14574; doi:10.3390/ijms 140714550

[4] R. Tietze, S. Lyer, S. Dürr, C. Alexiou, Nanoparticles for cancer therapy using magnetic forces, Nanomedicine (Lond), 7(3), (2012), 447-57

[5] Q.A. Pankhurst., N.K.T. Than., S.K. Jonhson, J. Dobson, Progress in applications of magnetic nanoparticles in biomedicine, J Phys D: Appl Phys., 42 (2009) 224001-224015

[6] S. Laurent, S. Dutz, U. Häfeli, M. Mahmoudi, Magnetic fluid hyperthermia: focus on superparamagnetic iron oxide nanoparticles, Adv. Colloid. Interface Sci. 166 (2011) 8-23

[7] R. Tietze, S. Lyer, S. Dürr, T. Struffert, T. Engelhorn, M. Schwarz, E. Eckert, T. Göen, S. Vasylyev, W. Peukert, F. Wiekhorst, L. Trahms, A. Dörfler, C. Alexiou, Efficient drug-delivery using magnetic nanoparticles--biodistribution and therapeutic effects in tumour bearing rabbits. Nanomedicine, 9(7), (2013) 961-971

[8] J.K. Oh, J.M. Park, Iron oxide-based superparamagnetic polymeric nanomaterials: Design, preparation, and biomedical application. Prog. Polym. Sci. 36 (2011) 168 - 189

[9] A.K. Andriola Silva, R. Di Corato, F. Gazeau, T. Pellegrino, C. Wilhelm, Magnetophoresis at the nanoscale: tracking the magnetic targeting efficiency of nanovectors, Nanomedicine, 7 (2012) 1713-1727

[10] J. Zhang, S. Xu, E. Kumacheva, Polymer Microgels: Reactors for Semiconductor, Metal and Magnetic Nanoparticles, J. Am. Chem. Soc., 126 (25), (2004) 7908-7914

[11] S.K. Suh, K. Yuet, D.K. Hwang, K.W. Bong, P.S. Doyle, T.A. Hatton, Synthesis of nonspherical superparamagnetic particles: in situ coprecipitation of magnetic nanoparticles in microgels prepared by stop-flow lithography, J. Am. Chem. Soc., 134 (2012) 7337-7343

[12] N. C. Bigall, C. Wilhelm, M.-L. Beoutis, M. García-Hernandez, A. A. Khan, C. Giannini, A. Sanchez-Ferrer, R. Mezzenga, M. E. Materia, M. A. Garcia, F. Gazeau, A. M. Bittner, L. Manna, T. Pellegrino, Colloidal Ordered Assemblies in a Polymer Shell - A Novel Type of Magnetic Nanobeads for Theranostic Applications, Chem. Mater. 25 (7), (2013) 1055-1062 
[13] K. Mosbach, L. Anderson, Magnetic ferrofluids for preparation of magnetic polymers and their application in affinity chromatography, Nature, 270 (1977) 259-261

[14] H. Shang, W.S. Chang, S. Kan, S.A. Majetich, G.U. Lee, Synthesis and characterization of paramagnetic microparticles through emulsion-templated free radical polymerization, Langmuir 22 (2006) 2516-2522

[15] P. Qiu, C. Jensen, N. Charity, R. Towner, C. Mao, Oil Phase Evaporation-Induced SelfAssembly of Hydrophobic Nanoparticles into Spherical Clusters with Controlled Surface Chemistry in an Oil-in-Water Dispersion and Comparison of Behaviors of Individual and Clustered Iron Oxide Nanoparticles, J. Am. Chem. Soc. 132 (2010) 17724-17732

[16] R. Turcu, I. Craciunescu, A. Nan, Magnetic Microgels: Synthesis and Characterization, in: H. Nirschl and K. Keller (Eds.) Upscaling of Bio-Nano-Processes, Lecture Notes in Bioengineering, DOI: 10.1007/978-3-662-43899-2_4, Springer-Verlag Berlin Heidelberg 2014, in press

[17] K. Landfester, Synthesis of Colloidal Particles in Miniemulsions, Annu. Rev. Mater. Res. 36 (2006) 231-279

[18] R. Tietze, E. Schreiber, S. Lyer, C. Alexiou, Mitoxantrone loaded superparamagnetic nanoparticles for drug targeting: a versatile and sensitive method for quantification of drug enrichment in rabbit tissues using HPLC-UV, Journal of biomedicine \& biotechnology. (2010) 597304

[19] L. E. Munoz, C. Maueröder, R. Chaurio, C. Berens, M. Herrmann, C. Janko, Colourful death: six-parameter classification of cell death by flow cytometry--dead cells tell tales, Autoimmunity 46(5) (2013) 336-341

[20] C. Paquet, H.W. de Haan, D.M. Leek, H.Y. Lin, B. Xiang, G. Tian, A. Kell, B. Simard, Clusters of Superparamagnetic Iron Oxide Nanoparticles Encapsulated in a Hydrogel: A Particle Architecture Generating a Synergistic Enhancement of the T2 Relaxation, ACS Nano 5 (2011) 3104-3112

[21] B. Luo, X.J. Song, F. Zhang, A. Xia, W.L. Yang, J.H. Hu, C.C. Wang, Multi-Functional Thermosensitive Composite Microspheres with High Magnetic Susceptibility Based on Magnetite Colloidal Nanoparticle Clusters, Langmuir 26 (2010) 1674-1679 
[22] D. Susan-Resiga, V. Socoliuc, T. Boros, T. Borbáth, O. Marinica, A. Han, L. Vékás, The influence of particle clustering on the rheological properties of highly concentrated magnetic nanofluids, J. Colloid Interface Sci., 373 (2012) 110-115

[23] O. Glatter, A new method for the evaluation of small-angle scattering data, J. Appl. Crystallogr. 10 (5) (1977) 415-421

[24] J. S. Pedersen, Analysis of small-angle scattering data from colloids and polymer solutions: modeling and least-squares fitting, Adv. Colloid Interface Sci. 70 (1997) 171-210

[25] P. W. Schmidt, Some Fundamental Concepts and Techniques Useful in Small-Angle Scattering Studies of Disordered Solids, in: H. Brumberger (Ed.) Modern Aspects of SmallAngle Scattering, Dordrecht: Kluwer Academic Publishers (1995) pp. 1-56

[26] G. Beamson, D. Briggs, High Resolution XPS of Organic Polymers, Ed. L. Baffins, Wiley: Chichester, England, 1992

[27] M. Descostes, F. Mercier, N. Thromat, C. Beaucaire, M. Gautier-Soyer, Use of XPS in the determination of chemical environment and oxidation state of iron and sulfur samples: constitution of a data basis in binding energies for $\mathrm{Fe}$ and $\mathrm{S}$ reference compounds and applications to the evidence of surface species of an oxidized pyrite in a carbonate medium, Appl. Surf. Sci. 165 (2000) 288-302 